\title{
Crisis y austeridad: amenaza para el empleo femenino en las regiones europeas
}

\author{
Crisis and austerity: threat for women's employment \\ in the European regions \\ Elena Martínez-Tola / elena.martineztola@ehu.es \\ https://orcid.org/0000-0001-8314-2925 \\ Ma.Luz de la Cal-Barredo/ luz.cal@ehu.es \\ https://orcid.org/000-0003-3893-2578 \\ Irantzu Álvarez-González / irantzu.alvarez@ehu.eus \\ https://orcid.org/0000-0003-4444-292X \\ Universidad del Pais Vasco/Euskal Herriko Unibertsitatea, España
}

\begin{abstract}
The objective of the study is to explore the relation between unemployment rates for men and women and the variation in public employment at regional level. To this end, a number of indicators are presented for the descriptive analysis of unemployment at regional level in the EU-15 and results are mapped. The main conclusion is that the marked sectoral segregation of employment by sex and the role of employment in the public sector for women should be taken into account when designing employment policies. On the contrary, employment growth in certain sectors and cuts in public spending in others introduce a bias that can boost male employment while delaying the recovery of feminine employment.
\end{abstract}

Key words: crisis, sectoral segregation by sex, European regions.

Resumen: El objetivo del trabajo es explorar la relación entre las tasas de paro de mujeres y de hombres, y la variación en el empleo público a escala regional. Para ello se plantean diversos indicadores orientados al análisis descriptivo del desempleo a nivel regional en el ámbito de la UE-15 y se hace una mapificación de los resultados. La principal conclusión es que la importante segregación sectorial del empleo por sexo y el papel que desempeña el empleo en el sector público en el trabajo femenino deben ser tomados en cuenta a la hora de diseñar las políticas laborales. De lo contrario, el fomento del empleo en ciertos sectores y/o los recortes de gasto público en otros incorporan un sesgo que puede estar impulsando el empleo masculino y retrasando la recuperación del femenino.

Palabras clave: crisis, desempleo femenino, segregación sectorial por sexo, regiones europeas. 


\section{Introducción}

Los efectos de la crisis económica y de las posteriores políticas de ajuste que se han implementado en el ámbito europeo no se han sentido de igual manera en todos los territorios ni en todos los grupos de población. Respecto a lo último, hay que señalar importantes diferencias por grupos de edad, por niveles de ingreso y también por sexo, que es el aspecto que abordamos en este trabajo.

A raíz de la crisis y en torno a las medidas de austeridad implementadas, ha surgido una relevante producción científica que explora los distintos impactos sufridos por hombres y mujeres. Se trata de trabajos que señalan la pertinencia de un análisis de género de las crisis, en concreto de la ya conocida como la Gran Recesión que se inició en 2008 (Smith y Villa, 2013; Rubery, 2015).

Las investigaciones se han ocupado, en primer lugar, de estudiar los impactos que la caída de la actividad económica tiene sobre el empleo masculino y sobre el femenino, aspecto que está profundamente mediatizado por la existencia de una fuerte segregación sectorial del empleo por sexo (Martínez-Tola y de la Cal, 2017). En segundo lugar, los análisis han abordado los efectos de las políticas económicas, fundamentalmente presupuestarias, bajo la óptica de género (Ginn, 2013).

El objetivo de este estudio es profundizar en el conocimiento de las tendencias en curso, de manera que sea posible identificar con mayor claridad la situación relativa en la cual se encuentran las regiones europeas respecto al desempleo femenino y masculino, después de haber sufrido el impacto de la crisis y las políticas de ajuste presupuestario instauradas en el ámbito europeo.

Se trata de un resultado relevante, pues deja en evidencia la necesidad de atender de forma pormenorizada los impactos regionales y de género de las políticas públicas más generales que, en muchas ocasiones, no hacen sino exacerbar las desigualdades preexistentes y dificultar en mayor medida el logro de sendas de recuperación estables a medio y largo plazo, así como los objetivos de igualdad de género en el ámbito laboral.

Para ello, en el segundo apartado se presentan las relaciones que la literatura establece entre la crisis económica, las políticas de austeridad y la evolución del desempleo en las economías europeas desde una perspectiva de género, para, en el tercer epígrafe, establecer las principales tendencias observables en el desempleo de mujeres y hombres en la escala regional para el periodo 2007-2014. 
En el cuarto apartado se analiza la brecha de género en el desempleo a partir de las tasas de paro masculina y femenina, avanzando una primera clasificación de las regiones en función del mayor o menor aumento de dichas tasas. En la quinta parte del trabajo se plantea de manera exploratoria el papel que ha tenido la evolución del empleo público regional en las diferencias observadas en el desempleo, para terminar con un apartado de conclusiones en el cual se recogen las principales ideas desarrolladas en este trabajo.

\section{Crisis, políticas de austeridad y sus efectos en el empleo}

Los análisis más generales realizados hasta la fecha hacen referencia a que la actual crisis podría estar afectando de manera diferente que las anteriores (la de principios de la década de 1980 y la de principios de 1990) al empleo femenino, dado que lo ha golpeado antes y con mayor severidad (Smith y Villa, 2013: 224; Martínez-Tola y de la Cal, 2017).

Se hace referencia a tres hipótesis clásicas para explicar estos diferentes impactos: la de sustitución de empleo femenino por masculino durante las crisis, por ser el primero más barato; la de segregación sectorial del empleo masculino y femenino que protege a las mujeres en las recesiones por estar concentradas en ciertos sectores; y la del efecto amortiguador, que remite a la mano de obra femenina entrando en el mercado laboral en las épocas de auge (trabajador adicional) y saliendo del mercado laboral en las de crisis (trabajador desanimado) (Humphries, 1988, citado en Smith y Villa, 2013).

Estos efectos se presentan de manera simultánea entre distintos grupos de mujeres y subsectores. Por países, se detectan diferencias en cuanto a su intensidad. Un reciente estudio para el caso de España e Italia (Addabbo $e t$ al., 2015) concluye que el efecto trabajador adicional es importante entre las trabajadoras españolas y que es mucho más significativo cuando la pareja está en desempleo o trabajando a tiempo parcial. En cambio, Italia es uno de los pocos países de la OCDE en los cuales el efecto trabajador desanimado es más fuerte que el de trabajador adicional entre las mujeres.

Con todo, estos recientes análisis referentes al impacto de la crisis en el empleo masculino y femenino (Smith y Villa, 2013) son más precisos que los antes mencionados en al menos dos aspectos. Primero, en el de señalar la importancia de una perspectiva de medio y largo plazo, y no centrarse solo en los impactos iniciales de la recesión. Ello ha revelado que en los primeros años de la crisis de 2008, como consecuencia del derrumbe de los sectores fuertemente masculinizados, el desempleo creció sobre todo entre los hombres; mientras que las mujeres estuvieron relativamente "protegidas", porque 
los sectores en los que de modo habitual trabajan no entraron en crisis en esos primeros años y por su mayor presencia en el empleo público.

Después, la crisis se extendió a todos los sectores económicos, afectando negativamente también al empleo femenino. Ello se acentuó todavía más con los recortes presupuestarios que contrajeron aún más la demanda y penalizaron el empleo público. La secuencia no es idéntica para todas las economías europeas, ya que algunas, como la irlandesa, sufrieron desde la primera etapa de la crisis (2008-2010) los recortes del gasto público y la congelación del empleo público (Barry y Conroy, 2013). A nivel mundial (Ortiz y Cummins, 2013), se habla de tres fases: la de expansión fiscal (2008-2009), la de inicio de la contracción fiscal (2010-2012) y la de intensificación de la contracción fiscal (2013-2015).

Segundo, en el de intentar avanzar cómo se producirá la recuperación del empleo una vez superada la crisis. En concreto, se trata de valorar cómo pueden ser las futuras transiciones desde el desempleo hasta el empleo, que bien podrían ser muy diferentes entre mujeres y hombres, e implicar para un número significativo de ellas un desempleo de larga duración (Martínez y de la Cal, 2017).

Otras líneas de trabajo interesantes son las que apuntan que los cambios en la regulación laboral se están llevando a cabo sin considerar sus efectos en la equidad de género en el mercado laboral. Es probable que muchos de los cambios que se han realizado reduzcan la protección de trabajadores vulnerables e incrementen la segmentación. En específico, el European Trade Union Institute ha señalado, entre estos cambios, la promoción de la descentralización de la negociación colectiva que da paso a una mayor dispersión salarial y a los descuelgues en los acuerdos colectivos; la reducción real e incluso nominal de los salarios mínimos; la promoción de la subcontratación en los servicios públicos, etcétera.

Además de reducirse la protección para los grupos peor situados en el mercado laboral, entre los cuales predominan las mujeres, se empeoran también las condiciones de trabajo de los grupos bien situados en el mercado laboral, como los empleados públicos, muchos de los cuales son mujeres (ETUI, 2014).

Como ya hemos dicho anteriormente, los análisis se han ocupado también de estudiar los efectos que la austeridad presupuestaria tiene sobre el empleo y el desempleo femeninos. La primera respuesta europea a la crisis financiera que se inició en 2008 confió en las recetas keynesianas, favoreciendo la expansión del gasto público para estabilizar la economía. Sin embargo, el rescate de los bancos por parte de los Estados con el consiguiente incremento 
del déficit público pronto precipitó a algunos de estos Estados a los denominados "rescates" y a procesos de consolidación fiscal.

Las políticas de austeridad han sido de obligado cumplimiento para dichos estados, pero en otros no rescatados se siguió este mismo ejemplo. Ello mostraría que tan sólo estamos asistiendo a un episodio más de la respuesta neoliberal a las crisis que nos acompañan desde la década de 1980, o como algunos trabajos la denominan, la versión austeritaria de las políticas deflacionistas (Gálvez y Rodríguez, 2013: 120).

Los análisis realizados señalan que las medidas de austeridad no están justamente distribuidas entre la población, que los hogares con menor nivel de ingreso y las personas de más edad resultan más afectados (Ginn, 2013). Se indican cuatro grandes roles del Estado social cuya contracción es especialmente significativa para las mujeres y para el progreso de la igualdad de género (Rubery, 2015): el Estado como fuente de apoyo a la renta; como proveedor de servicios públicos gratuitos o subsidiados; como empleador directo; y como protección contra la mercantilización de la sociedad. Cada uno de estos grandes roles o áreas de intervención estatal tienen efectos directos e indirectos en el empleo y en el trabajo de las mujeres, tema que nos ocupa en este artículo.

En cuanto al papel del Estado como fuente de renta, los efectos inmediatos que tienen los recortes en subsidios por hijos, desempleo o vejez en el conjunto de la población se intensifican en el caso de las mujeres. La razón no es otra más que esta: el modelo de empleo femenino existente con más parcialidad, menor remuneración, interrupciones en la carrera profesional, etcétera implica una mayor dependencia de las mujeres a las prestaciones y subsidios, y, por ello, a una mayor vulnerabilidad ante los recortes en el gasto social (Martínez y de la Cal, 2017).

Para el caso de Reino Unido, se destaca que los recortes en prestaciones de la asistencia social afectan más a las mujeres, pues entre ellas es mucho más frecuente el cobro de estas prestaciones porque su nivel de ingresos es inferior al de los hombres (se refieren al subsidio para la calefacción y al de alquiler de vivienda). Como resultado, las familias monoparentales (de las cuales el 90\% está encabezada por mujeres) y las pensionistas solas constituyen los dos grupos que más han visto reducida su renta media anual en 2010 (Ginn, 2013).

La reducción de los servicios públicos que soportan la reproducción social puede implicar una intensificación del trabajo femenino, fundamentalmente del no remunerado. El incremento del trabajo no remunerado en los hogares, combinado con el desigual reparto de las tareas domésticas y de cuidado entre mujeres y hombres, provoca que un buen número de mujeres se retire 
del mercado laboral durante las crisis (Gálvez y Rodríguez, 2012). Además de afectarlas como usuarias de los servicios públicos, los recortes en los relacionados con educación, salud y servicios sociales también las afectan como empleadas en ellos, dado que un elevado porcentaje del empleo femenino está en el sector público.

El papel del sector público como empleador promueve la igualdad de género, lo cual se da por el aumento de la participación laboral de las mujeres y por la reducción de la brecha salarial de género. Además, esto ha contribuido al ascenso de categoría del empleo femenino. Recientes análisis (Grimshaw et al., 2012) para el caso de cinco países europeos ${ }^{1}$ muestran que, a consecuencia de la mayor concentración de las mujeres trabajadoras en el sector público, es probable que las medidas de austeridad tengan un efecto desproporcionado en el empleo femenino.

La protección frente a la mercantilización de la sociedad y la preservación de la esfera de lo público son vistas como necesarias para avanzar en la igualdad de género. Se considera que la austeridad supone una amenaza a la esfera de lo público, porque ataca a las aspiraciones del Estado de bienestar, por los recortes en recursos y porque ha reinventado los bienes y valores públicos como mercancías (Rubery, 2015).

Cabe destacar una serie de trabajos que estudian el retroceso en materia de género e igualdad durante los años de la crisis. Para el caso de la economía española, esto se produciría por la acción combinada de los recortes en el presupuesto público, el retroceso en las leyes en materia de igualdad y la eliminación de organismos de igualdad. Se puede señalar también la diferente incidencia de estos procesos según la orientación ideológica del partido en el gobierno (Paleo y Alonso, 2014).

En una línea muy similar, el estudio de Barry y Conroy (2013), para el caso de Irlanda, habla del desmantelamiento de estructuras establecidas para la promoción de la igualdad o su absorción en otros departamentos gubernamentales, así como de recortes presupuestarios en medidas con el fin de promover la igualdad para las mujeres entre 2008 y 2010. Para algunas autoras, como Rubery (2015), sin embargo, la erosión de las políticas en pro de la igualdad de género había comenzado antes de la crisis. En concreto, se habla de su pérdida de visibilidad desde el año 2000 y de su desaparición desde 2003 como uno de los cuatro pilares de la Estrategia de Empleo Europea (Rubery, 2015).

Una serie de trabajos apunta a que los efectos de la crisis sobre la equidad de género pueden ir más allá de los económicos y laborales. Específicamente,

1 Entre el 67 y el 74\% para el caso de Suecia, Reino Unido, Alemania, Hungría y Francia. 
la reprivatización de los cuidados, como consecuencia de los recortes en los servicios públicos, puede suponer una relativa "vuelta a casa" para las mujeres como herramienta clave de sumisión y control social (Gálvez y Rodríguez, 2013). Ello iría de la mano con una cierta reorientación de la agenda de igualdad, en la cual la promoción de la maternidad pasaría a ocupar un papel central.

Para el caso español, se detecta que algunas nuevas leyes de igualdad autonómicas se enmarcan en la protección de la maternidad y el apoyo a las mujeres gestantes, focalizando su atención en el rol de la mujer como madre; mientras que los varones están prácticamente ausentes. Ello representa un giro copernicano respecto a leyes de igualdad anteriores que habían evolucionado hacia conceptos de igualdad de naturaleza relacional, lo cual implicaba la transformación de los roles de ambos sexos (Paleo y Alonso, 2014: 56).

Desde el punto de vista sindical, se alerta de que los asuntos de equidad de género caigan de las mesas de negociación colectiva en tiempos de austeridad. Al tiempo, se señala que la equidad puede funcionar como cuña para sostener un renacimiento de la negociación colectiva innovadora, y, por extensión, una revitalización y renovación del sindicalismo (Briskin, 2014).

Finalizaremos este breve recorrido haciendo referencia a los enfoques que apuntan hacia la necesidad de un análisis y una implementación de políticas sensibles al género, algo que desafortunadamente no ha existido en la crisis. La secuencia y la incidencia de la crisis son diferentes para mujeres y hombres; por lo tanto, un enfoque de este tipo tiene mucho que ofrecer en la recuperación del empleo tanto para las mujeres como para los hombres (Bettio y Verashchagina, 2013; Villa y Smith, 2010: 53).

\section{El desempleo en las regiones europeas}

En los últimos años se ha podido identificar un proceso de convergencia en las tasas de desempleo dentro de la mayoría de los países europeos, en un contexto marcado por un aumento generalizado del desempleo. No obstante, algunas regiones han mostrado tendencias positivas en términos de creación de empleo y crecimiento, lo cual ha llevado a que, a escala europea, se haya producido una mayor divergencia en términos agregados, dando lugar a tensiones entre los países más y menos afectados por la crisis (Crescenzi et al., 2016).

Así, aunque el contraste entre los países europeos del centro y la periferia son notables, existen importantes diferencias también dentro de los países. 
En este sentido, el centro de Portugal, el sur de España e Italia, la zona central de Grecia, la antigua Alemania del este y el norte de Inglaterra y Escocia han sido especialmente golpeados por la crisis en términos de destrucción de empleo. Por otro lado, el norte de Italia, la zona de Madrid y el norte de España, el sudeste del Reino Unido, el sur de Alemania y las tres capitales nórdicas han respondido de mejor manera que sus respectivos países a la crisis (Fratesi y Rodríguez-Pose, 2016).

En términos generales, el impacto de la crisis en materia de empleo ha hecho que las diferencias de género tiendan a disminuir, aunque el ajuste se haya realizado en un sentido no deseado (Bettio y Sansonetti, 2015). Esto se debe a que los sectores productivos más afectados en un primer momento por la crisis son algunos de los más masculinizados; el aumento de la tasa de paro para los hombres ha hecho que ésta se acerque a las tasas de las mujeres, tradicionalmente superiores. No obstante, las distintas características de los territorios hacen que las realidades regionales, después de un periodo de siete años tras la irrupción de la crisis, no sean homogéneas a nivel europeo (Addabbo et al., 2015: 504-505).

Según Eurostat (2007 y 2014), la tasa de paro en la UE-15 ha aumentado en 3,5 puntos porcentuales (pasando de un 7 a un 10,5\%): para los hombres el aumento medio ha sido de 4 puntos porcentuales (del 6,4 al 10,4\%) y para las mujeres de 2,8 puntos (del 7,8 al 10,6\%). De esta forma se confirma la tendencia a un acercamiento de las tasas de paro, aunque el desempleo femenino era y sigue siendo mayor que el masculino, y se ha dado en un contexto marcado por el aumento del desempleo en ambos casos.

El desempleo femenino ha aumentado sustancialmente en las regiones de los países periféricos, algunas de las cuales ya partían de tasas especialmente elevadas; en el año 2014 destacaban los altos valores en las regiones griegas y del sur de España e Italia. Para el caso de los hombres, partiendo de unas tasas de paro menores que las femeninas en la mayor parte de los casos, en 2014 las mayores tasas se encontraban en las regiones del sur de España, siendo también altas en el resto de las regiones españolas, en las griegas y en el sur de Italia. Cabe destacar la mejoría experimentada por algunas regiones del norte de Alemania, cuyas tasas de desempleo descienden de manera relevante en el periodo considerado.

El escenario regional en el año 2014 para las tasas de paro refleja una mayor similitud entre la situación de hombres y mujeres, al tiempo que se identifica una zona central con tasas de paro reducidas, un primer anillo de regiones con situaciones más diversas aunque marcadas por menores tasas 
de paro y una tercera dimensión, formada por las regiones españolas, griegas y del sur de Italia, en las cuales las tasas de paro son especialmente elevadas.

En una primera fase de la crisis (periodo 2007-2010), la tasa de paro de las mujeres aumentó de forma significativa en las regiones españolas e irlandesas. En una segunda fase (2010-2014), los mayores aumentos en dicha tasa se produjeron en las regiones griegas, el sur de Italia y Holanda. Así, para el conjunto del periodo estudiado, las tasas de paro han experimentado un mayor aumento en las regiones españolas (sobre todo en las orientales), griegas, italianas, irlandesas y holandesas.

Las mayores reducciones en la tasa de paro se encuentran en las regiones alemanas, belgas y austriacas. En el caso de la tasa de paro de los hombres, en la primera fase el aumento del desempleo afectó también muy notablemente a las regiones españolas e irlandesas, y en un segundo periodo a las griegas e italianas. En el periodo 2007-2014, las regiones más afectadas por el incremento del desempleo masculino fueron las de España, Grecia y el norte de Italia (véanse mapas $1,2,3$ y 4$){ }^{2}$

Uno de los resultados de las tendencias antes descritas muestra que la relación entre la tasa de paro de hombres y de mujeres en las regiones europeas se ha hecho más estrecha. ${ }^{3}$ Para el primer año, en el $77 \%$ de las regiones las tasas de las mujeres eran superiores a las de los hombres; mientras que ese porcentaje se reduce hasta el $50 \%$ para 2014. Aunque este resultado contribuye a ofrecer una idea general de que las desigualdades laborales de género tienden a disminuir - pues también sucede con la convergencia en términos de tasa de ocupación y, para muchos países, en términos salariales-, en la mayoría de las regiones dicha confluencia se experimenta en un contexto marcado por un aumento del desempleo y una precarización de las condiciones laborales.

Así, la agenda de la igualdad de género corre el riesgo de pasar a un segundo plano mientras los esfuerzos se centren en la recuperación del empleo masculino, que, en caso de producirse, se basará en trabajos con condiciones más precarias que las de los destruidos durante la crisis (OCDE, 2012).

2 Los mapas y las tablas se encuentran en el Anexo, al final del presente artículo (Nota del editor).

3 De hecho, en el año 2007 el coeficiente de correlación entre ambas era de 0,664 ( $p<0,001)$ y en el año 2014 pasa a ser del 0,958 ( $p<0,001)$, lo que indica una aproximación entre ambas tasas en el ámbito regional. 


\section{Relación entre el desempleo femenino y masculino en las regiones de la UE-15}

Por lo general, la brecha de género para la tasa de paro se define como la diferencia entre la tasa de las mujeres y la tasa de los hombres, y se expresa en puntos porcentuales como unidad de medida. En la mayoría de los casos, hasta épocas muy recientes, esta brecha solía dar lugar a un resultado positivo, en tanto el desempleo de las mujeres era mayor que el de los hombres y se medía en puntos porcentuales.

En el presente estudio se procede a plantear las diferencias de género en este ámbito en términos relativos; por lo que el ratio o brecha de género en este caso se calculará como el cociente entre la tasa de paro de las mujeres y la de los hombres. De esta manera siempre se obtendrá un resultado positivo, y la unidad representará la igualdad de tasas de paro; mientras que los mayores a 1 indican un mayor desempleo femenino que masculino para la región considerada.

Este indicador enfatiza la relación entre ambas tasas, lo cual permite una mejor interpretación de su evolución en el tiempo. Su principal problema es que puede darse la situación de percibirse una mejoría en términos de brecha de género en un contexto donde las tasas de desempleo sean superiores para ambos grupos, aunque esto también sucede con la brecha calculada en términos de diferencia de tasas. Asimismo, da lugar a situaciones en las cuales se plantea una brecha similar para regiones con valores absolutos de desempleo muy diferentes, lo cual hace necesario analizar siempre este tipo de indicadores de manera conjunta con los valores absolutos para llevar a cabo una interpretación más ajustada a la realidad.

Las regiones griegas, italianas y españolas presentan un mayor ratio o brecha de género tanto en 2007 como en 2014, aunque se confirma la tendencia a su reducción a lo largo del tiempo. En el otro extremo, para 2014, en muchas regiones la brecha de género se situó en valores menores a 1 , lo cual vendría a mostrar que la tasa de paro de los hombres supera a la de las mujeres, como es el caso de todas las regiones finlandesas, irlandesas y buena parte de las danesas, suecas y alemanas.

En 2007, las mayores diferencias se encontraron en varias regiones griegas, donde la tasa de paro de las mujeres es hasta cuatro veces superior a la de los hombres. Asimismo, se halla en este grupo la región italiana de Veneto, cuya brecha es también muy elevada pero para unos niveles de la tasa de paro mucho más reducidos. 
En el otro extremo, es decir, con brechas de género reducidas, encontramos regiones del Reino Unido y Alemania, donde la tasa de paro de los hombres es hasta dos veces superior a la de las mujeres, manteniéndose ambos en niveles bajos para el caso de las regiones británicas (Merseyside, Hampshire e Isle of Wight, East Yorkshire y Northern Lincolnshire, Cheshire, Greater Manchester, South Yorkshire, Tees Valley y Durham). En el caso de las regiones alemanas (Bremen y Berlín), estos niveles son especialmente elevados tanto para hombres como para mujeres.

Tras la crisis económica y las políticas de ajuste podemos observar que la situación es marcadamente diferente. La media de la tasa de paro de las mujeres se sitúa en torno al 10,5\%, registrando valores mínimos en la región alemana de Oberpfalz (2,3\%) y el máximo en la región española de Andalucía $(37,3 \%)$. Por su parte, la media de la tasa de paro de los hombres se ubica en el 9,8\%, marcando su máximo también en Andalucía (32,7\%) y su mínimo en Unterfranken, Alemania (2,5\%). La brecha de género se sitúa en torno a 1 de media (igualdad de tasas), siendo su máximo en la región italiana de Veneto $(1,7)$ y su mínimo en Dorset and Somerset, Reino Unido $(0,55)$.

Para el año 2014, en el ranking de las regiones con mayores brechas de género en la tasa de paro siguen apareciendo una mayoría de regiones griegas, aunque para este año nos encontramos también con la región italiana de Véneto en el primer puesto. Cabe destacar que las regiones de otros países con elevadas brechas de género en las tasas de paro presentan unos niveles de tasa de paro muy inferiores a los de las regiones griegas.

Entre las regiones con menor brecha de género en 2014 se hallan mayoritariamente las británicas, donde la tasa de paro masculina es mayor que la femenina. También entre las diez señaladas se encuentra la región holandesa de Zeeland y la alemana Münster.

El ratio o brecha de género para la tasa de paro ha descendido en el $86 \%$ de las regiones consideradas (y ha aumentado en un total de 28 casos); aunque esta relativa mejoría puede producirse de diversas maneras (véanse Tabla 1 y Mapa 5). En la mayor parte de los casos (116 regiones), la menor distancia se debe a que la tasa de desempleo masculino se ha incrementado en mayor medida que el femenino, y sería la situación de las regiones españolas, italianas, la mayoría de las portuguesas, francesas, suecas, danesas e irlandesas.

Sin embargo, también puede destacarse el caso de las 38 regiones donde la brecha o ratio de género disminuye en un contexto marcado por el descenso de las tasas de paro (en este caso, la reducción de la tasa femenina es mayor que la masculina, lo cual lleva a su convergencia). En dicho caso se encuentran casi todas las regiones alemanas; por lo tanto, puede considerarse una realidad 
particular de esta economía. Por último, entre las regiones donde se reducen las diferencias de género, cabe señalar la existencia de un grupo formado por 19 regiones pertenecientes a distintos países (Reino Unido, Francia, Austria y Bélgica), en las que se registra un descenso de la tasa de paro femenina y un aumento de la masculina.

Por otro lado, encontramos 28 regiones donde la tasa de paro de las mujeres se aleja de la masculina en este periodo de siete años, debido a que ha aumentado en mayor medida la tasa de las mujeres que la de los hombres. Se trata principalmente de regiones británicas, aunque encontramos ejemplos de este tipo en Francia, Portugal, Dinamarca y Alemania. En este sentido, cabe señalar que representan el $14 \%$ de los territorios analizados y existe un fuerte componente nacional, ya que 20 de ellas pertenecen al Reino Unido.

Por todo ello, se puede decir que existe una tendencia general en este periodo hacia un acercamiento de las tasas de paro de mujeres y hombres en las regiones europeas en su conjunto, aunque el elemento diferencial estará relacionado con la manera como se produce: por un aumento en las tasa de paro para ambos sexos o debido a una mayor disminución en las tasas de paro femeninas que en las masculinas. Entonces, si excluimos del análisis las regiones británicas - que presentan una variedad de situaciones y ciertas características específicas-, se puede comprobar que hay una tendencia general a la reducción de la brecha en las tasas de desempleo, aunque es preciso diferenciar dos claros escenarios.

Por una parte, hallamos las regiones del centro de Europa, donde en los últimos años han descendido las tasas de paro, y, por tanto, la convergencia se ha dado en un contexto favorable. Por otra, encontramos las regiones periféricas en las que los ajustes se han producido de manera menos deseable, con aumentos de las tasas de paro, en algunos casos muy considerables, tanto para hombres como para mujeres. No obstante, aún en el primero de los casos resultaría imprescindible ahondar en el estudio de las condiciones laborales de los empleos actuales.

\section{Relación entre la evolución del empleo público y la brecha de género en el desempleo}

Uno de los principales argumentos que explican el impacto que ha tenido la crisis en el empleo de hombres y mujeres es el de la segregación laboral, entendida como la mayor concentración del empleo en distintos sectores y, en particular, en el empleo público. Así, a partir del año 2010 se comienzan a poner en marcha en los países europeos las políticas de austeridad, con el 
ajuste presupuestario que conllevan, lo cual desemboca en diferentes situaciones según el país del que se trate. Por ello, en este apartado se analiza el periodo 2010-2014, en el que el ajuste en términos de empleo público comienza a manifestarse.

Puedecomprobarsequeel retrocesoen elempleopúblicoesmayoren Grecia $(-13,6 \%)$, España $(-4,2 \%)$ y Holanda $(-5,6 \%)$; mientras que en Dinamarca, Irlanda e Italia se produce un estancamiento del número de personas empleadas. Para el caso de las mujeres, los principales retrocesos ocurren en Grecia, Holanda, España y Dinamarca; mientras que para los hombres, las peores cifras se registran en Grecia, Holanda, España, Bélgica y Portugal (Tabla 2).

Las diferencias regionales dentro de los países en la evolución del empleo público son considerables, aunque destaca por su magnitud el retroceso sufrido en las regiones griegas y holandesas, seguidas por algunas españolas e italianas.

Al considerar la clasificación de las regiones presentada en el apartado anterior, en las regiones donde la brecha de género aumenta (la mayoría británicas), el empleo público se ha incrementado en una media de $2,2 \%$; y en las regiones donde esta brecha ha disminuido, el empleo público ha aumentado una media del $1,5 \%$. Si nos centramos en este segundo grupo con menor brecha de género, en las regiones en las que aumentan las tasas de paro (tipo 2), el empleo público ha retrocedido en una media de $0,7 \%$; mientras que en las regiones donde ha descendido el desempleo (tipo 4), la ocupación en el sector público se ha incrementado en $6,7 \%$.

Las regiones pertenecientes al tipo 6 (reducción de la tasa femenina y aumento de la masculina) experimentan un incremento del 3,6\% en el empleo público entre 2010 y 2014 . Aunque con este análisis no es posible establecer una relación temporal y causal entre estas circunstancias, las tendencias observadas apuntan a que en las regiones más perjudicadas por la crisis se comprueba una reducción o estancamiento del empleo público que, sin duda, contribuye a ahondar el problema laboral existente. Además, en la medida en que este tipo de ocupación supone un nicho laboral de empleo de calidad para muchas mujeres, las alternativas laborales para ellas serán aún menores en el caso de profundizarse en este sentido desde los gobiernos regionales, nacionales y europeos.

La relación entre el aumento de la tasa de paro femenina en el periodo 2010-2014 y la variación del empleo público es notable y de signo negativo. ${ }^{4}$

4 El coeficiente de correlación entre la variación de la tasa de paro de las mujeres y del empleo público en el periodo 2010-2014 muestra un valor de $-0,569$ ( $\mathrm{p}<0,001)$. 
Las regiones con mayor desempleo y recortes de empleo público son la mayoría de las griegas, italianas, holandesas y españolas. También las regiones francesas donde los descensos en los empleos públicos han sido de mayor importancia presentan considerables aumentos de las tasas de paro para las mujeres (véase Mapa 6).

La relación entre el aumento de la tasa de paro de los hombres y la variación del empleo público también es relevante, aunque algo menos fuerte que para el caso de las mujeres, y además presenta una relación inversa (véase Mapa 7). ${ }^{5}$ Destaca la situación en la que se encuentran las regiones griegas, españolas y algunas italianas, belgas, holandesas y francesas, donde los recortes en el empleo público han sido especialmente duros.

El mayor impacto del ajuste en el empleo público recae sobre las mujeres no sólo porque en general su presencia es mayoritaria en el mismo, sino porque existen diversos elementos que inciden en este ajuste. La segregación horizontal que se observa también en el empleo público (mayor presencia de mujeres en sanidad, educación o servicios sociales, por ejemplo), su menor representación en los altos cargos, así como sus diferentes relaciones contractuales (ocupan también una mayoría de los puestos no funcionariales), provoca que sean especialmente afectadas de manera negativa por dichos ajustes (OCDE, 2012).

A modo de resumen, puede decirse que los datos para las regiones europeas confirman que el ajuste realizado en el empleo público en el periodo 2010-2014 está relacionado de manera más fuerte con el aumento del desempleo femenino que con el masculino. Así, en Grecia y Holanda, en la mayoría de las regiones se ha producido una reducción del empleo público que ha ido acompañada de un aumento muy importante de las tasas de paro de hombres y mujeres.

En el caso de las regiones españolas, aunque la destrucción del empleo público no ha sido tan significativo como en Grecia, los aumentos de desempleo en las economías regionales sí han sido muy elevados. Para el caso de Alemania y Reino Unido, existe una gran variedad de situaciones en términos de aumento o reducción de empleo público, para una situación de reducción de tasas de desempleo.

5 El coeficiente de correlación entre la variación de la tasa de paro de las mujeres y del empleo público en el periodo 2010-2014 muestra un valor de -0,500 ( $\mathrm{p}<0,001)$. 


\section{Conclusiones}

En los últimos años, tras el estallido de la crisis económica y financiera en 2008 , son numerosos los indicadores que muestran una convergencia en las condiciones laborales de hombres y mujeres, aunque estos resultados deben ser contextualizados para hacer una adecuada interpretación de los procesos en curso.

En este sentido, se constata un acercamiento de las tasas de paro en las regiones europeas aunque existen diferencias importantes por destacar. En términos generales, se puede hablar de una realidad polarizada en la que las regiones de la periferia europea siguen el patrón más habitualmente citado de cierres de brecha de género para la tasa de paro, debido a un mayor aumento del desempleo masculino que del femenino; mientras que en las regiones del centro de Europa (mayoritariamente alemanas, pero también francesas, austriacas y belgas), el cierre de las brechas se produce por un importante retroceso en las tasas de desempleo femeninas.

Así, aunque en muchos casos la brecha en términos de tasa de empleo entre mujeres y hombres se ha reducido, ello no es un resultado positivo en términos de equidad de género, sino que refleja, en gran medida, el rápido empeoramiento de la situación de los hombres.

A pesar de que estos resultados contribuyen a ofrecer una idea general de que las desigualdades laborales de género tienden a disminuir - pues también sucede con la convergencia en términos de tasa de ocupación y, para muchos países, en términos salariales-, en la mayoría de las regiones esta confluencia se experimenta en un contexto marcado por un aumento del desempleo y una precarización de las condiciones laborales.

De esta manera, es posible que los objetivos de la agenda de igualdad de género en las condiciones laborales pasen a ocupar una posición subordinada o desaparezcan dentro de las prioridades políticas de los gobiernos, para dar paso a iniciativas centradas en la promoción de las oportunidades de empleo en sectores masculinizados. Este planteamiento resulta especialmente inadecuado, sobre todo si se tiene en cuenta que el ajuste en el empleo femenino ha podido aún no terminar y las oportunidades de empleo de las mujeres y sus condiciones, ya de por sí más precarias, pueden verse mermadas en los próximos años de forma notable.

En esta línea, es preciso abogar por el análisis pormenorizado con perspectiva de género de los efectos de la crisis y de las medidas de ajuste a medio y largo plazo en el empleo de las mujeres y hombres, que pongan en evidencia 
las tendencias subyacentes reales y sirvan de base para el diseño de las políticas económicas en general, y de empleo en particular, a poner en marcha por las diferentes escalas de gobierno.

En dicho contexto, la evolución del empleo público conforma un área de estudio de especial relevancia, en la medida que representa un nicho de empleo de calidad para las mujeres y habrá de determinar no sólo sus posibilidades de ocupación en términos cuantitativos, sino también las condiciones laborales relacionadas con sus entradas y salidas del mercado de trabajo.

Los recortes presupuestarios y su impacto en la reducción del empleo público no son homogéneos a escala europea, aunque la mayoría de países ha puesto en marcha políticas en esta dirección a partir del año 2010. Será preciso realizar un seguimiento detallado de los efectos que las mismas han tenido sobre la vida de hombres y mujeres. En este sentido, en el periodo 2010-2014 se pueden apreciar algunas pautas diferenciales en el empleo público, aunque los ajustes podrán observarse con mayor fidelidad en los próximos años.

Una primera aproximación al tema nos ofrece un panorama general que confirma el mayor impacto que tiene sobre la tasa de paro de las mujeres la variación del empleo en el sector público para las regiones europeas. Destaca la situación de las regiones griegas, holandesas, españolas e italianas, donde las reducciones en el tamaño del empleo público han venido acompañadas de elevadas tasas de desempleo femenino. Puede interpretarse también que los mayores ajustes se han producido en las economías más afectadas por la crisis, de forma que el problema del aumento del desempleo no estaría ligado solamente a esta circunstancia.

No obstante, el recorte de empleo público no parece que haya ayudado a solucionar esta situación, sino al contrario, habría contribuido a agravarlo y afecta en mayor medida a las mujeres. Este mayor impacto estaría provocado no sólo porque ellas son las principales ocupadas en sectores públicos como los de educación, sanidad, servicios sociales, etc., los cuales han sido objeto de fuertes recortes presupuestarios en los últimos años, sino también porque dentro de la administración pública su posición es más frágil que la de los hombres, ocupando en mayor medida puestos con menor categoría y mayor temporalidad.

Ligado a lo anterior, las transiciones del empleo al desempleo también son de vital importancia dentro de este análisis, pues existe la posibilidad de que al reducirse las posibilidades de empleo en general, y de empleo de calidad en el sector público en particular, las mujeres encuentren aún más reducidas sus posibilidades de conseguir un empleo de calidad y se amplíen 
todavía más las diferencias de género en algunos indicadores de calidad del empleo, que deberán, a su vez, ser objeto de especial estudio y consideración.

Una futura línea interesante consistiría en sistematizar las características de las regiones, de acuerdo a cómo ha sido su comportamiento en cuanto a la evolución de la brecha en la tasa de paro de hombres y mujeres durante la crisis. Se trataría de explorar si la ampliación y/o reducción de la brecha de género en la tasa de paro durante obedece a los cambios en el número de empleos públicos. Trabajos previos (Elomäki, 2012; Leschke y Jepsen, 2012) ya han constatado que algunas de las políticas de austeridad, en concreto las de recortes de personal o de salarios en sectores donde el peso de las mujeres es importante (como la sanidad o la educación), tienen un impacto negativo en cuanto a la equidad de género en diferentes países de la UE.

Se trataría de constatar si se puede llegar a esta misma conclusión para algunos de los mercados laborales regionales y también para conocer qué características tienen estos mercados. La distribución de los efectos negativos de la crisis posee componentes espaciales importantes, como ya han señalado algunos estudios anteriores. Concretamente, parece ser que dichos efectos negativos se han dejado sentir más en las regiones de la UE y de Estados Unidos económicamente más débiles y con alta concentración de empleos públicos (Kitson et al., 2011).

La mayor dependencia de este tipo de empleos para las mujeres supondría que los efectos de las políticas de austeridad tendrían un componente sectorial-espacial, el cual implicaría también un componente de género claramente desfavorable para las mujeres.

\section{Referencias}

Addabbo, Tindara et al. (2015), "Gender differences in labor force participation rates in Spain and Italy under the Great Recession”, en Revista de Economia Mundial, núm. 41, España: Sociedad de Economía Mundial.

Barry, Ursula y Conroy, Paula (2013), "Ireland in crisis 2008-2012, Women, austerity and inequality". Disponible en: http://hdl.handle.net/10197/4820 [5 de abril de 2017].

Bettio, Francesca y Verashchagina, Alina (2013), "Women and men in the great European recession", en Karamessini, M. y Rubery, J. [eds.], Women and Austerity. The Economic Crisis and the Future for Gender Equality, USA: Routledge.

Bettio, Francesca y Sansonetti, Sylvia (2015), "Visions for gender equality", en European Union. Disponible en: http://ec.europa.eu/justice/gender-equality/files/documents/ vision_report_en.pdf [ 4 de abril de 2017].

Briskin, Linda (2014), "Austerity, union policy and gender equality bargaining", en Transfer: European Review of Labour and Research, vol. 20, núm.1. Disponible en: http:// journals.sagepub.com/doi/abs/10.1177/1024258913515161 [5 de enero de 2017]. 
Crescenzi, Riccardo et al. (2016), "The geography of the economic crisis in Europe: national macroeconomic conditions, regional structural factors and short-term economic performance", en Cambridge Journal of Regions, Economy and Society, vol. 9, núm.1, Reino Unido: Cambridge Political Economy Society.

Elomäki, Anna (2012), “The price of austerity. The impact on women's rights and gender equality in Europe”. Disponible en: http://www.womenlobby.org/IMG/pdf/the price_of_austerity_-_web_edition.pdf [30 de mayo de 2017].

ETUI (2014), "Benchmarking working Europe". Disponible en: https://www.etui.org/ Publications2/Books/Benchmarking-Working-Europe-2014 [18 de abril de 2017].

Eurostat (2007), "Unemployment rates by sex, age and NUTS 2 regions (\%)", en Labour Force Survey database. Disponible en: http://ec.europa.eu/eurostat/data/database [5 de octubre de 2017].

Eurostat (2010), "Unemployment rates by sex, age and NUTS 2 regions (\%)", en Labour Force Survey database. Disponible en: http://ec.europa.eu/eurostat/data/database [5 de octubre de 2017].

Eurostat (2014), "Unemployment rates by sex, age and NUTS 2 regions (\%)", en Labour Force Survey database. Disponible en: http://ec.europa.eu/eurostat/data/database [5 de octubre de 2017].

Fratesi, Ugo y Rodríguez-Pose, Andrés (2016), “The crisis and regional employment in Europe: what role for sheltered economies?”, en Cambridge Journal of Regions, Economy and Society, vol. 9, núm.1, Reino Unido: Cambridge Political Economy Society.

Gálvez, Lina y Rodríguez, Paula (2012), "La desigualdad de género en las crisis económicas”, en Investigaciones Feministas, núm. 2, España: Universidad Complutense de Madrid.

Gálvez, Lina y Rodríguez, Paula (2013), "El empleo de las mujeres en la España democrática y el impacto de la Gran Recesión”, en Revista Internacional de Ciencias sociales, núm. 32. Disponible en: http://revistas.um.es/areas/article/view/192321 [12 de octubre de 2017].

Ginn, Jay (2013), "Austerity and inequality. Exploring the impact of cuts in the UK by gender and age", en Research on Aging and Social Policy, vol.1, núm. 1. Disponible en: http://www.hipatiapress.info/hpjournals/index.php/rasp/article/view/491 [12 de octubre de 2017].

Grimshaw, Damian et al. (2012), "Public sector pay and procurement in Europe during the crisis: the challenges facing local government and the prospects for segmentation, inequalities and social dialogue", en European Commision Project. VS/2011/0141. Disponible en: https://www.escholar.manchester.ac.uk/uk-ac-man-scw:239494 [12 de octubre de 2017].

Kitson, Michael et al. (2011), “The geographies of austerity”, en Cambridge Journal of Regions, Economy and Society, vol. 4, núm. 3, Reino Unido: Cambridge Political Economy Society.

Leschke, Janine y Jepsen, Maria (2012), "Introduction: Crisis, policy responses and widening inequalities in the EU”, en International labour review, vol. 151, núm.4, Suiza: Organización Internacional del Trabajo.

Martínez-Tola, Elena y de la Cal Barredo, María Luz (2017), “Una aproximación territorial y de género al desempleo durante la crisis. El caso de los municipios vascos", en Lurralde, núm. 40. Disponible en: http://www.ingeba.org/lurralde/lurranet/lur40/40martinez. pdf [30 de mayo de 2017]. 
OCDE (2012), "Cerrando las brechas de género: es hora de actuar". Disponible en: http:// www.sitioswwweb.com/miguel/Cerrando_las_brechas_de_g__nero.pdf [5 de mayo de 2017].

Ortiz, Isabel y Cummins, Matthew (2013), "The age of austerity: a review of public expenditures and adjustment in 181 countries. Initiative for policy dialogue and the south centre". Disponible en: https://papers.ssrn.com/sol3/papers.cfm?abstract id $=2260771$ [30 de mayo de 2017].

Paleo, Natalia y Alonso, Alba (2014), “¿Es solo una cuestión de austeridad? Crisis económica y políticas de género en España”, en Investigaciones feministas, núm. 5, España: Universidad Complutense de Madrid.

Rubery, Jill (2015), "Austerity, the public sector and the threat to gender equality", en The Economic and Social Review, vol. 46, núm. 1. Disponible en: http://www.esr.ie/article/ view/289 [12 de julio de 2017].

Smith, Mark y Villa, Paola (2013), "Recession and recovery", en Bettio, Francesca et al. [eds.], Gender and the European labour market, Inglaterra: Routledge.

Villa, Paola y Smith, Mark (2010), "Gender equality, employment policies and the crisis in the EU Member States", en EU Expert group on gender and employment. Syntesis Report. Disponible en: file:///C:/Users/Mari\%20Luz/Downloads/Gender-employmentcrisis\%20(2).pdf [12 de julio de 2017].

\section{Anexo}

\section{Tabla 1}

\section{Tipología de regiones}

\begin{tabular}{lcc}
\hline & Mayor brecha & Menor brecha \\
\hline Aumento tasa de paro mujeres y aumento & 19 regiones & 116 regiones \\
\cline { 2 - 3 } tasa de paro hombres & (Tipo 1) & $($ Tipo 2) \\
\hline $\begin{array}{l}\text { Disminución tasa de paro mujeres y } \\
\text { disminución tasa de paro hombres }\end{array}$ & 2 regiones & 38 regiones \\
\hline $\begin{array}{lcc}\text { Aumento tasa de paro mujeres y } \\
\text { disminución tasa de paro hombres }\end{array}$ & (Tipo 3) & $($ Tipo 4) \\
\hline $\begin{array}{l}\text { Disminución tasa de paro mujeres y } \\
\text { aumento tasa de paro hombres }\end{array}$ & (Tipo 5) & - \\
\hline & 2 regiones & 19 regiones \\
\hline
\end{tabular}

Fuente: Elaboración propia con base en datos de Eurostat (2007 y 2010). 


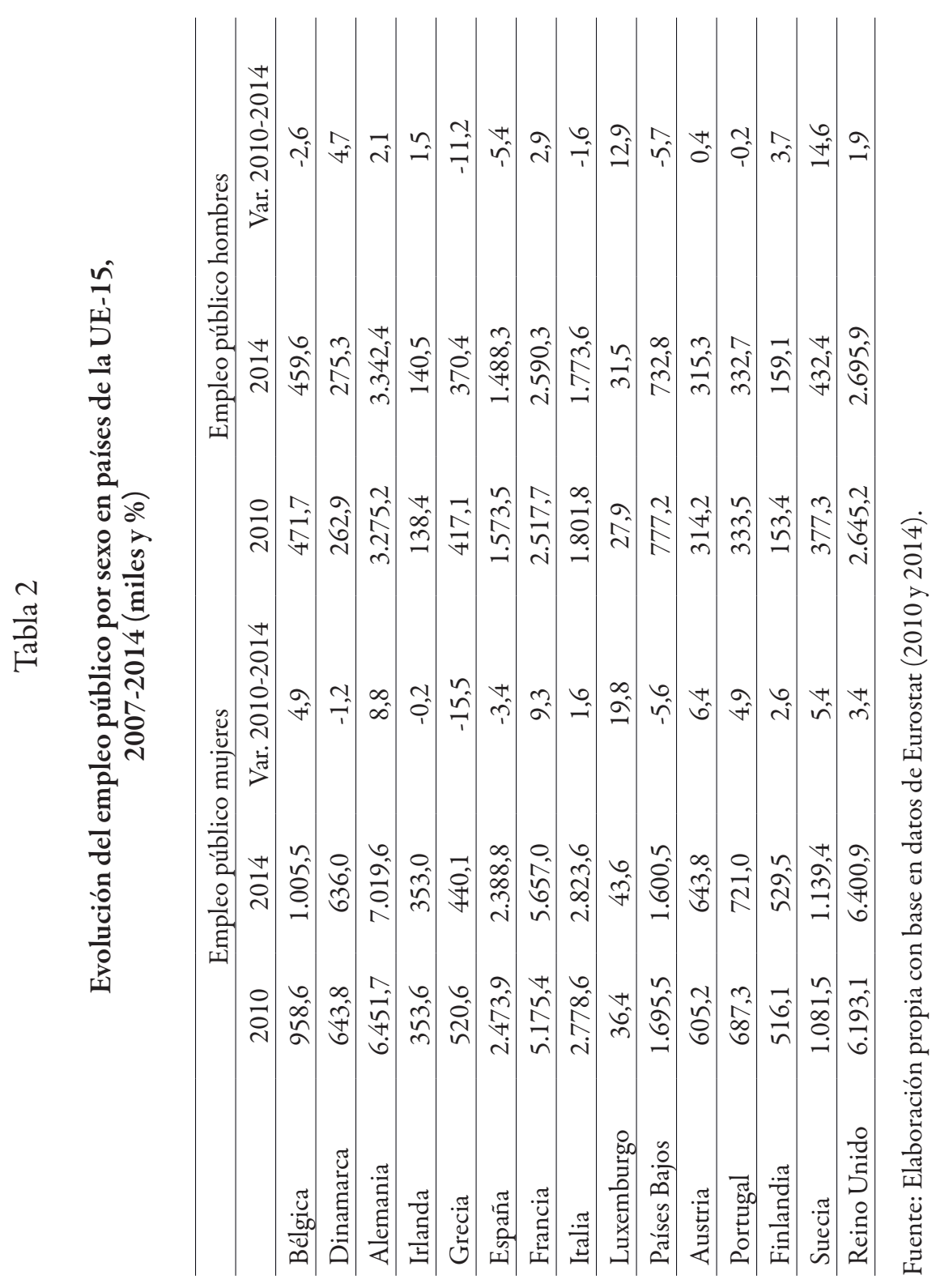




\section{Mapa 1}

Variación en las tasas de paro de los hombres entre 2007 y 2010
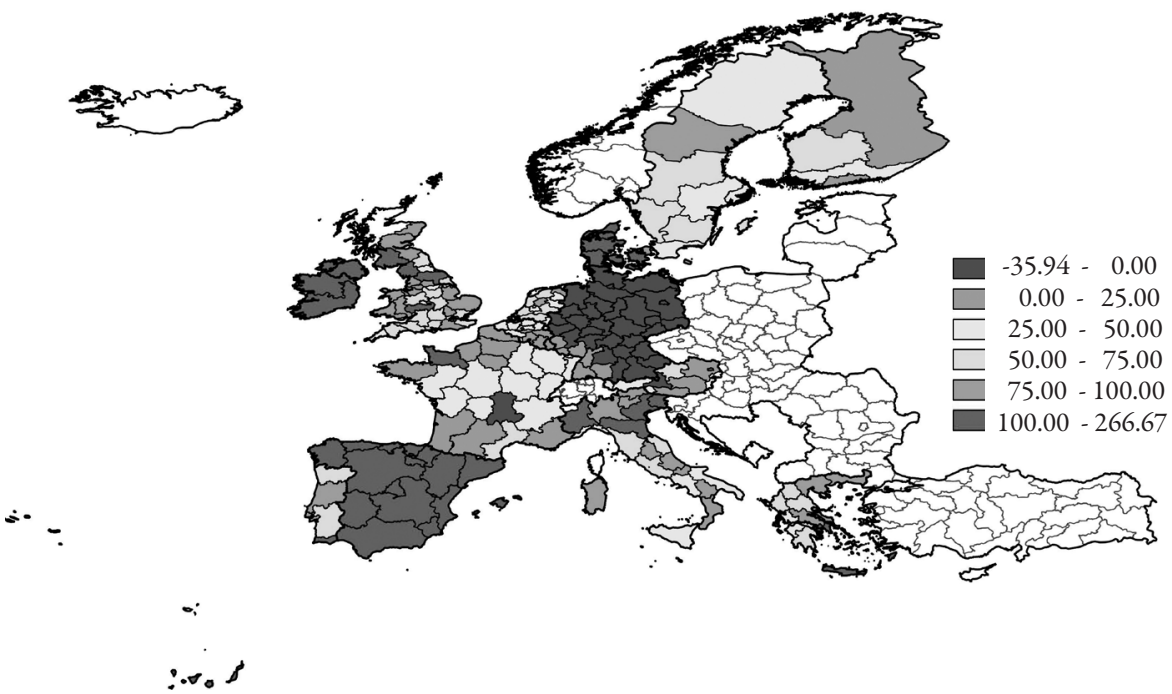

Fuente: Elaboración propia con base en datos de Eurostat (2007 y 2010).

\section{Mapa 2}

Variación en las tasas de paro de los hombres entre 2010 y 2014
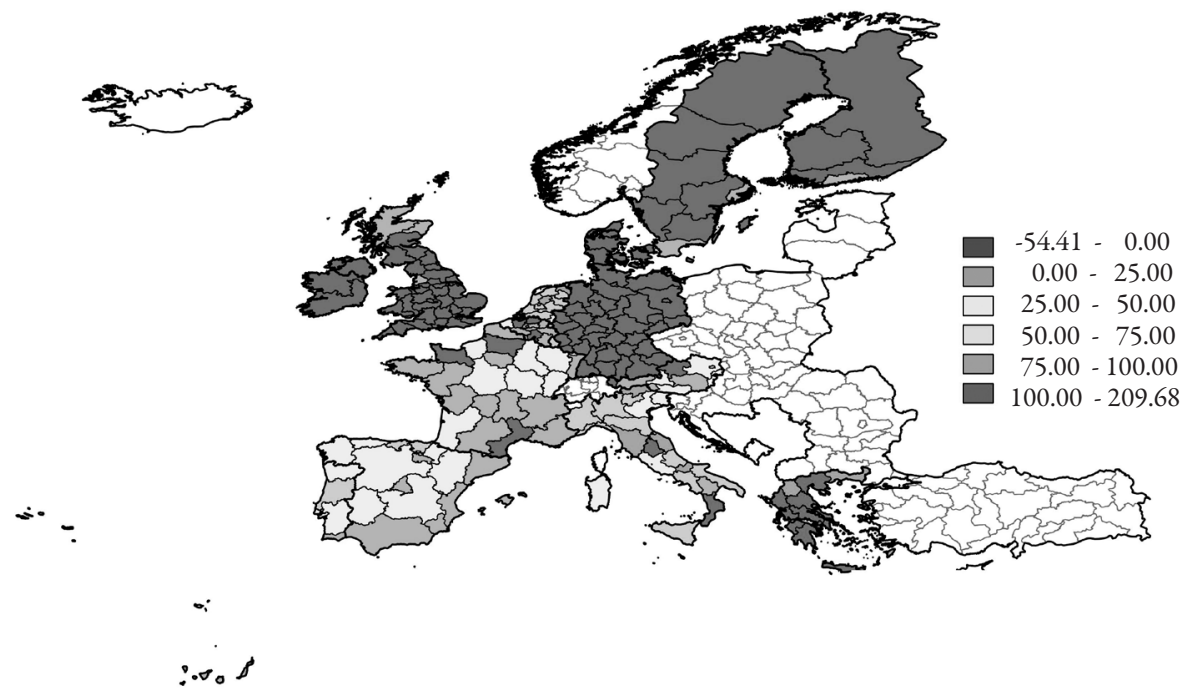

Fuente: Elaboración propia con base en datos de Eurostat (2010 y 2014). 


\section{Mapa 3}

Variación en las tasas de paro de las mujeres entre 2007 y 2010

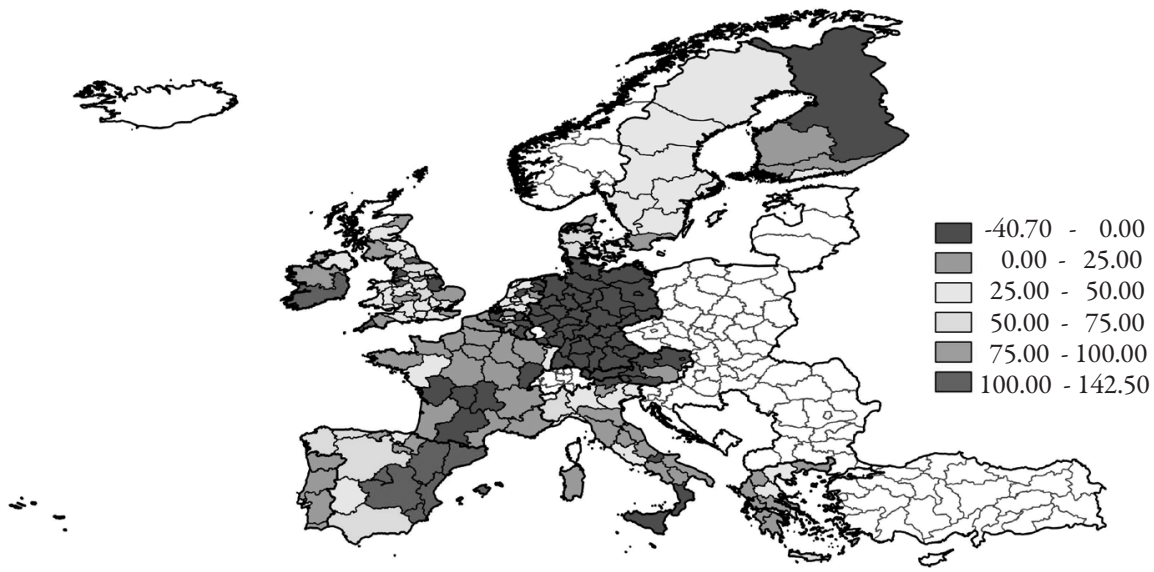

Fuente: Elaboración propia con base en datos de Eurostat (2007 y 2010).

\section{Mapa 4}

Variación en las tasas de paro de las mujeres entre 2010 y 2014

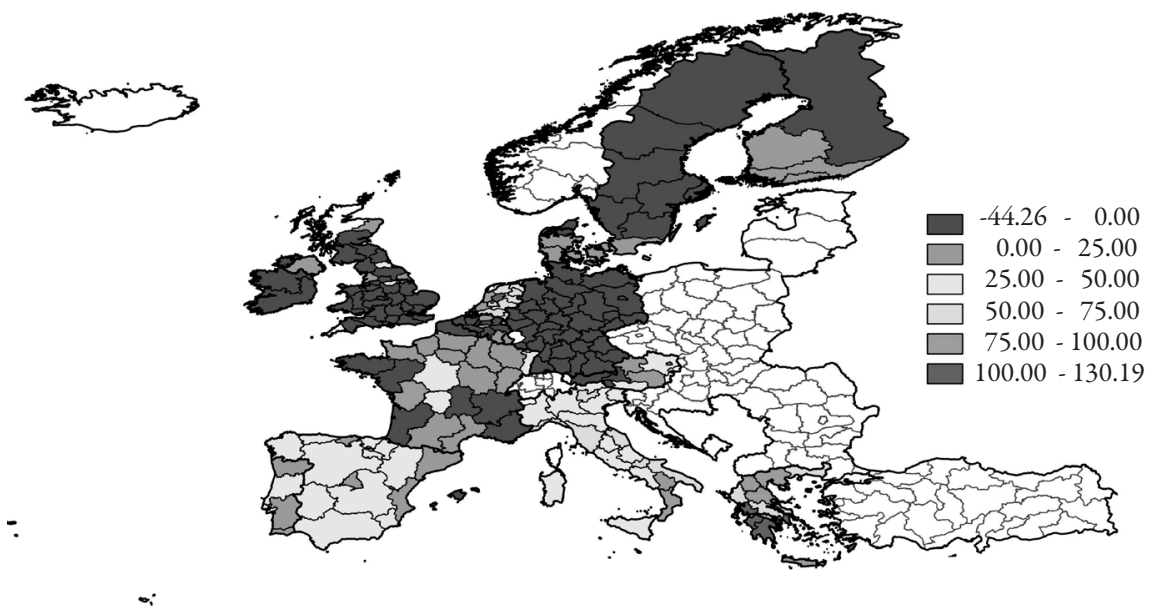

Fuente: Elaboración propia con base en datos de Eurostat (2010 y 2014). 


\section{Mapa 5}

Tipología de las regiones de la UE-15 según el ratio/brecha de género

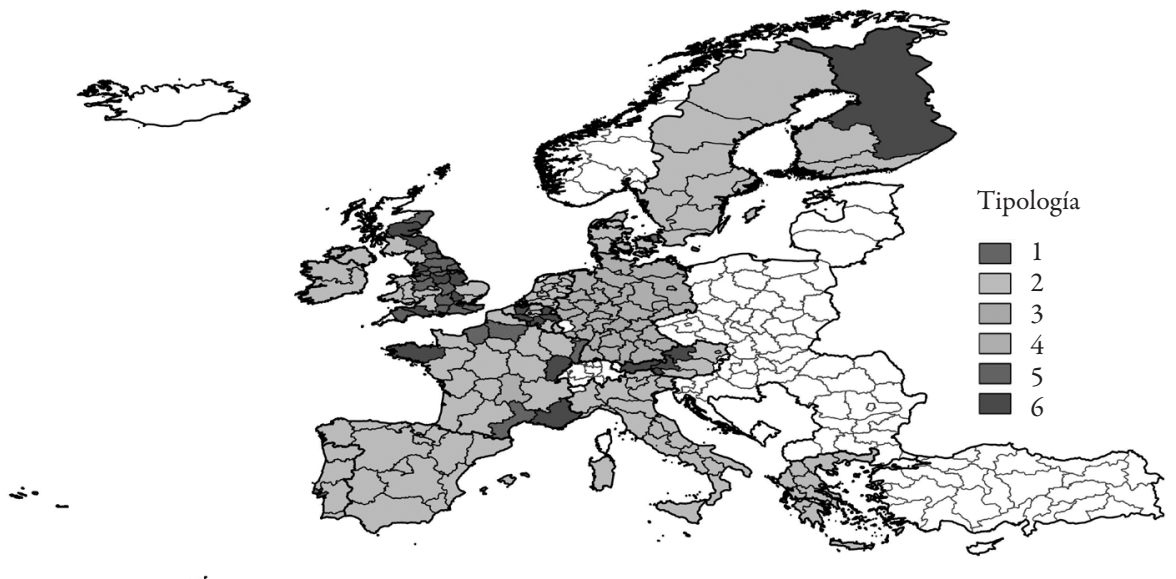

Fuente: Elaboración propia en base a datos de Eurostat (2007 y 2010).

\section{Mapa 6}

Clasificación de regiones según la relación entre la variación en la tasa de paro de las mujeres y la variación en el empleo público (2010-2014)
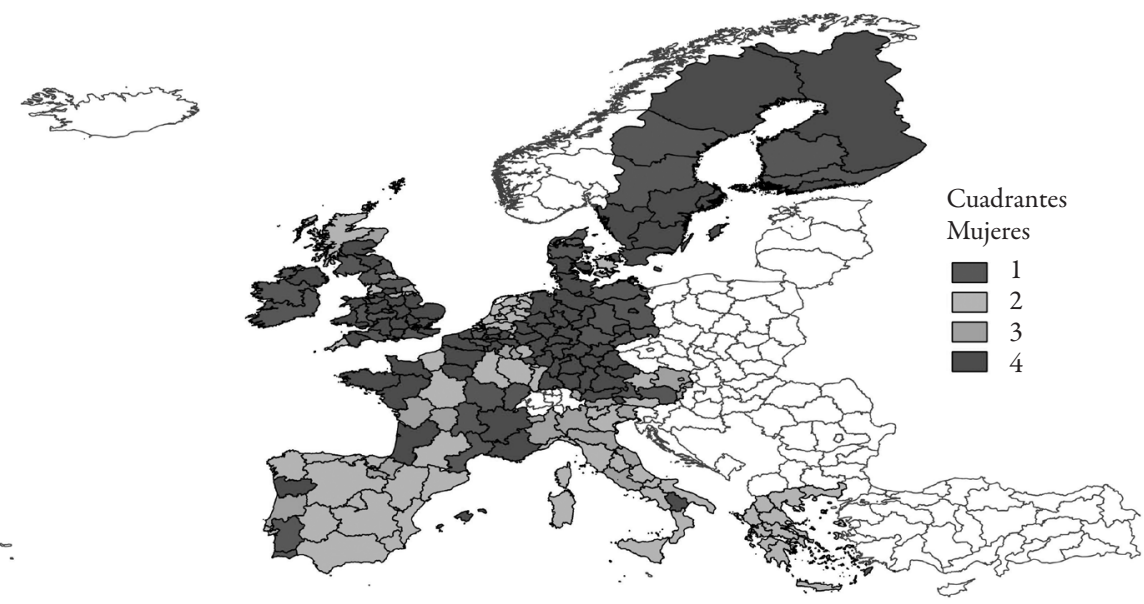

Fuente: Elaboración propia en base a datos de Eurostat (2010 y 2014). 


\section{Mapa 7}

Clasificación de regiones según la relación entre la variación en la tasa de paro de los hombres y la variación en el empleo público (2010-2014)

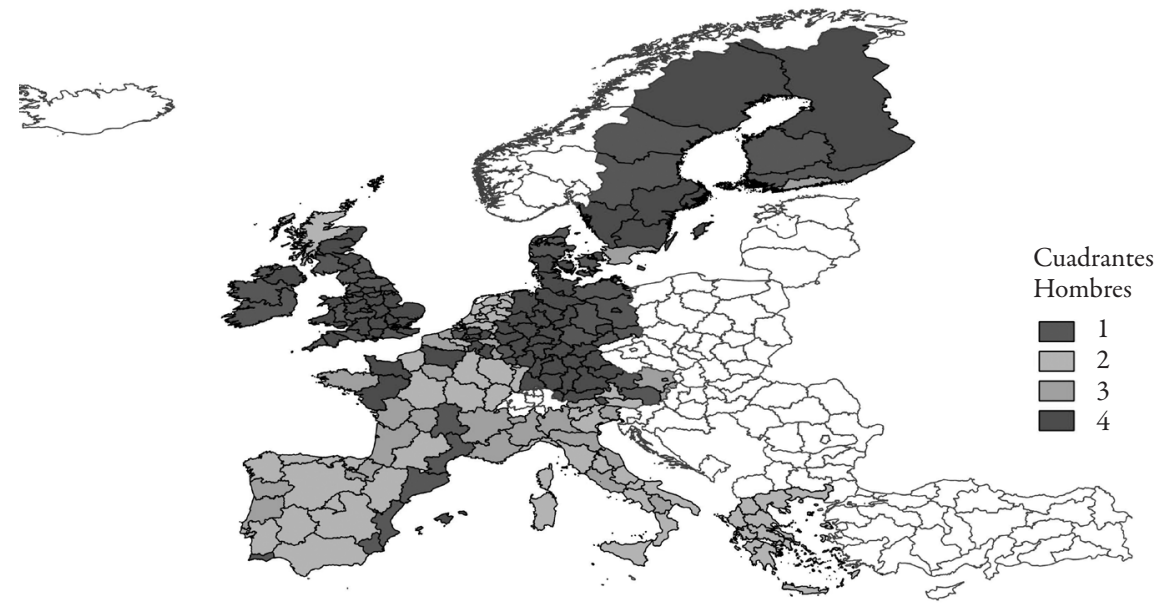

Fuente: Elaboración propia con base en datos de Eurostat (2010 y 2014).

Elena Martínez-Tola. Doctora en Ciencias Económicas. Profesora Agregada en la Facultad de Economía y Empresa de la Universidad del País Vasco/ Euskal Herriko Unibertsitatea, en el Departamento de Economía Aplicada desde el año 2000. Líneas de investigación: economía feminista y desarrollo regional y urbano. Publicaciones recientes: Martínez, Elena y de la Cal, Ma. Luz, "Una aproximación territorial y de género al desempleo durante la crisis. El caso de los municipios vascos”, en Lurralde, núm. 40 (2017); Larrañaga, Mertxe y Martínez, Elena, "Desigualdades económicas de género en la Comunidad Autónoma del País Vasco", en Ekonomiaz, núm. 91 (2017); Zurbano, Mikel, Bidaurratzaga, Eduardo y Martínez, Elena, "Las transformaciones de los modelos territoriales de desarrollo en el contexto de la globalización. Aportaciones desde la perspectiva del desarrollo humano local", en Revista de Estudios Regionales, núm. 99 (2014).

Ma. Luz de la Cal-Barredo. Doctora en Ciencias Económicas. Profesora Titular en la Facultad de Relaciones Laborales y Trabajo Social de la Universidad del País Vasco/Euskal Herriko Unibertsitatea, en el Departamento de Economía Aplicada desde 1990. Líneas de investigación: pobreza, mercado 
laboral y economía feminista. Publicaciones recientes: Martínez Tola, Elena y de la Cal Barredo, Ma. Luz, "Una aproximación territorial y de género al desempleo durante la crisis. El caso de los municipios vascos", en Lurralde, núm. 40 (2017); Gómez, Mikel y de la Cal, Ma. Luz, "Posibles conflictos éticos entre los paradigmas científico-tecnológicos dominantes y la mejora del bienestar de la población en algunas economías emergentes", en Revista de Economía Mundial, núm. 35 (2013); de la Cal, Ma. Luz, "Realidad y retos de la protección social en Euskadi”, en Ekonomiaz, núm. 81 (2012).

Irantzu Álvarez-González. Doctora por la Universidad del País Vasco/ Euskal Herriko Unibertsitatea y profesora en el Departamento de Expresión Gráfica y Proyectos de Ingeniería de la Escuela de Ingeniería de la misma universidad. Líneas de investigación: aplicaciones de los Sistemas de Información Geográfica, análisis espacial, ordenación del territorio. Publicaciones recientes: Esteban, M., Álvarez, I. y Torres, M. C., "Políticas de suelo industrial en Álava”, en Boletín de la Asociación de Geógrafos Españoles, núm. 58 (2012); Álvarez, I., Esteban, M. y Torres, M. C., "Sistema de Información Geográfica Industrial: una herramienta para la gestión y la promoción de la industria. El caso de Álava (País Vasco)", en Boletín de la Asociación de Geógrafos Españoles, núm. 76 (2016); Álvarez, I. y Moreno, J., "La Torca del Carlista en el karst de ranero. La cueva de Pozalagua”, en La Torca del Carlista: uno de los mayores volúmenes subterráneos del mundo, Leitzaran, Sua Edizioak (2016).

Recepción: 29 de junio de 2017.

Aprobación: 7 de marzo de 2018. 
